\title{
Association between Cystatin C and MRI Measures of Left Ventricular Structure and Function: Multi-Ethnic Study of Atherosclerosis
}

\author{
Subhashish Agarwal,, ${ }^{1}$ Vinay Thohan, ${ }^{1}$ Michael G. Shlipak, ${ }^{2}$ Joao Lima, ${ }^{3}$ David A. Bluemke, ${ }^{4}$ \\ David Siscovick, ${ }^{5}$ Antoinette Gomes, ${ }^{6}$ and David M. Herrington ${ }^{1}$ \\ ${ }^{1}$ Department of Cardiology, Wake Forest University, Winston-Salem, NC 27157, USA \\ ${ }^{2}$ University of California, San Francisco, CA 94143, USA \\ ${ }^{3}$ Division of Cardiology, John Hopkins University, Baltimore, MD 410-516-8000, USA \\ ${ }^{4}$ Division of Cardiology, John Hopkins University and Imaging Sciences Training Program, National Institutes of Health and National \\ Institute of Biomedical Imaging and Bioengineering, Bethesda, MD 20892, USA \\ ${ }^{5}$ Cardiovascular Health Research Unit, University of Washington, Seattle, WA 98195-5502, USA \\ ${ }^{6}$ UCLA David Geffen School of Medicine, CA 90095, USA
}

Correspondence should be addressed to Subhashish Agarwal, sa1972@gmail.com

Received 15 June 2011; Revised 23 July 2011; Accepted 26 July 2011

Academic Editor: Kazunari I. Kaneko

Copyright (C) 2011 Subhashish Agarwal et al. This is an open access article distributed under the Creative Commons Attribution License, which permits unrestricted use, distribution, and reproduction in any medium, provided the original work is properly cited.

Introduction. Reduced kidney function, approximated by elevated cystatin C, is associated with diastolic dysfunction, heart failure, and cardiovascular mortality; however, the precise mechanism(s) that account for these relationships remains unclear. Understanding the relationship between cystatin $\mathrm{C}$ and subclinical left ventricular (LV) remodeling, across ethnically diverse populations, may help explain the mechanisms underlying the association of kidney dysfunction with heart failure and cardiovascular mortality. Methods. Measures of cystatin C and LV parameters were obtained from the multi-ethnic study of atherosclerosis (MESA) cohort at baseline ( $N=4$, 970 with complete data on cystatin C and LV parameters). LV parameters; LV end-diastolic (LVEDV) and end-systolic volumes (LVESV), LV mass (LVM), concentricity (LV mass/LV end-diastolic volume), and LV ejection fraction (LVEF) were measured using magnetic resonance imaging. Nested linear models were used to examine the relationship between higher quartiles of cystatin C and LV parameters, with and without adjustment for demographics, height, and weight, and traditional cardiovascular risk factors. Similar analyses were performed stratified by ethnicity and gender. Results. A fully adjusted model demonstrated a linear relationship between higher quartiles of cystatin C and lower LVEDV, (Mean \pm SE, $128 \pm 0.7,128 \pm 0.7,126 \pm 0.7$, $124 \pm 0.8 \mathrm{~mL} ; P=0.0001)$. Associations were also observed between higher quartiles of cystatin C and lower LVESV $(P=0.04)$ and concentricity $(P=0.0001)$. In contrast, no association was detected between cystatin C and LVM or LVEF. In analyses stratified by race and gender, the patterns of association between cystatin $\mathrm{C}$ quartiles and LV parameters were qualitatively similar to the overall association. Conclusion. Cystatin C levels were inversely associated with LVEDV and LVESV with a disproportionate decrease in LVEDV compared to LVM in a multi-ethnic population. This morphometric pattern of concentric left ventricular remodeling, may in part explain the process by which kidney dysfunction leads to diastolic dysfunction, heart failure and cardiovascular mortality.

\section{Introduction}

Elevated cystatin C, a marker of reduced kidney function, is associated with increased cardiovascular mortality [1] and incident heart failure [2-4]. Cystatin $\mathrm{C}$ has been shown to be a superior marker of renal function compared to creatinine or estimated GFR as it is less affected by age, gender, and ethnicity [5-8]. Moreover, higher levels of cystatin C have been shown to be associated with increased LV mass [9], concentric LV hypertrophy $[9,10]$, and diastolic dysfunction [11]. An association between cystatin $\mathrm{C}$ and heart failure, particularly diastolic heart failure, is not unexpected given that it is a sensitive and specific marker of renal insufficiency and highly correlates with hypertension [9]; however, various 
lines of evidence suggest that the relationship between cystatin C and LV mass and hypertrophy may go beyond simple confounding due to its association with hypertension [11]. Data are needed to better define the relationship between cystatin $C$ and ventricular remodeling, which may clarify its relationship with heart failure and cardiovascular mortality and elucidate new mechanisms which may lead to development of novel therapies. Further, this will clarify the process by which mild-moderate kidney dysfunction may lead to ventricular remodeling and heart failure. We hypothesize that cystatin $\mathrm{C}$ is a marker for ventricular remodeling, independent of blood pressure, in a multi-ethnic population.

\section{Materials and Methods}

2.1. Study Population. The Multi-Ethnic Study of Atherosclerosis (MESA) design has been previously described [12]. Briefly, MESA is a prospective cohort study that began in July 2000 to investigate the prevalence, correlates, and progression of subclinical CVD. The study included 6814 racially diverse men and women aged 44-84 years old recruited from 6 US communities (Baltimore, Md; Chicago, Ill; Forsyth County, NC; Los Angeles County, Calif; northern Manhattan, NY; and St. Paul, Minn). MESA cohort participants were $38 \%$ Caucasian $(n=2,622), 28 \%$ African-American $(n=1,893), 22 \%$ Hispanic $(n=1,496)$, and $12 \%$ Chinese $(n=803)$. Individuals with a history of physician-diagnosed myocardial infarction, angina, heart failure, stroke, or transient ischemic attack, or who had undergone an invasive procedure for CVD (coronary artery bypass graft, angioplasty, valve replacement, pacemaker placement, or other vascular surgeries) were excluded from the study at baseline (20002002). This study was approved by the Institutional Review Boards of each study site, and written informed consent was obtained from all participants.

2.2. Laboratory Measures and Data Collection. Medical history, anthropometric measurements, and laboratory data for the present study were taken from the first examination of the MESA cohort (July 2000-August 2002). Information about age, sex, ethnicity, and medical history was obtained by questionnaires. Resting blood pressure was measured using Dinamap Monitor PRO 100 (Critikon, Tampa, Fl) automated oscillometric device. Three measurements were obtained at 1-minute intervals with the subject in the seated position with back and arm supported after $5 \mathrm{~min}$ of rest with an appropriate-sized cuff, with the cuff at the level of the heart, using a standardized protocol. The average of the second and third measurements was recorded as the resting blood pressure. Hypertension was defined as a systolic blood pressure $\geq 140 \mathrm{~mm} \mathrm{Hg}$, a diastolic blood pressure $\geq 90 \mathrm{~mm} \mathrm{Hg}$, or currently taking medications for blood pressure control. Smoking use was defined as never, former, and current smokers. Diabetes was defined as a fasting glucose $\geq 126 \mathrm{mg} / \mathrm{dL}$ or use of insulin or hypoglycemic medications. Total cholesterol was measured from blood samples obtained after a 12-hour fast and measured using a standardized kit (Roche Diagnostics). Cystatin C was measured from frozen sera at a central laboratory (University of Vermont, Colchester, Vt) using a BNII nephelometer (Dade Behring Inc, Deerfield, Ill) and a particle-enhanced immunonephelometric assay ( $\mathrm{N}$ Latex Cystatin C; Dade-Behring) [13]. The analytical coefficient of variation for this assay is $2.5 \%$.

\subsection{Magnetic Resonance Imaging Protocol. The MESA MRI} protocol has been described in detail elsewhere [14]. Briefly, cardiac MRI testing was performed at MESA study sites using a standard protocol and read at a central site (Johns Hopkins University, Baltimore, MD). LVEDV and LVESV, LVM and LVEF were determined using 1.5-Tesla MR scanners: Signa LX and CVi (GE Medical Systems, Waukesha, Wis) and Symphony and Sonata (Siemens Medical Systems, Erlangen, Germany). MRI was performed with a 4-element phasedarray surface coil placed anteriorly and posteriorly, with electrocardiogram gating and brachial artery blood pressure monitoring. Imaging consisted of cine images of the left ventricle with a temporal resolution of 50 milliseconds or less. LVEDV and LVESV were calculated using Simpson's rule (the summation of areas on each separate slice multiplied by the sum of slice thickness and image gap). LVM was determined by the sum of the myocardial area (the difference between endocardial and epicardial contour) times slice thickness plus image gap in the end-diastolic phase multiplied by the specific gravity of myocardium $(1.05 \mathrm{~g} / \mathrm{mL})$. LVEF was calculated as LV stroke volume divided by LVEDV multiplied by 100 . Concentricity was determined by obtaining a ratio of LVM by LVEDV. The interobserver variability in estimating LV parameters was: LVEDV, technical error of the mean (TEM\%, 95\% CI), $(4.4 \mathrm{~mL}, 95 \%$ CI $2.6,6.6)$; LVESV ( $12.8 \mathrm{~mL}, 95 \%$ CI 9.3, 16.2); LVM (6.0 gm, 95\% CI, 4.6, 7.4); LVEF $(5.1 \%, 95 \%$ CI $3.6,6.7)$ and intraobserver variability in estimating LV parameters was: LVEDV $(5.1 \mathrm{~mL}, 95 \% \mathrm{CI}$, 3.94, 6.15); LVESV (10.5 mL, 95\% CI, 8.17, 12.68); LVM (6.3 gm, 95\% CI, 5.17, 7.38); $\operatorname{LVEF}(3.9 \%, 95 \%$ CI, 3.06, 4.72) [14].

2.4. Statistical Methods. Differences in baseline characteristics were compared across quartiles using analysis of variance (ANOVA) for continuous variables and Chi-square tests for categorical variables. The LV parameters of interest, LVEDV, LVESV, LVM, LVEF, and concentricity (LVM/LVEDV), were compared across cystatin C quartiles using ANOVA.

Multivariate linear regression analyses in nested models were performed to evaluate the association of cystatin C quartiles, with the following outcomes: LVEDV, LVESV, LVM, LVEF, and concentricity (LVM/LVEDV). After univariate analysis (Model 1), the following covariates were considered as potential confounders: age, gender, race/ethnicity, height, and weight (Model 2); Model 2 covariates plus traditional cardiovascular risk factors: history of diabetes, antidiabetic medications, hypertension, systolic blood pressure, total cholesterol, antilipid medications, and smoking (Model 3). Similar linear analyses in nested models were performed stratified by ethnicity and gender. Interaction terms using (cystatin $\mathrm{C}$ quartile $\times$ race) and (cystatin $\mathrm{C}$ quartile $\times$ gender) were introduced in the model. 
TABLE 1: Baseline characteristics of 4,970 MESA participants by quartile of cystatin C.

\begin{tabular}{|c|c|c|c|c|c|}
\hline Variables & Quartile 1 & Quartile 2 & Quartile 3 & Quartile 4 & $P$ value \\
\hline \multicolumn{6}{|c|}{ Demographics } \\
\hline$(n=4,970)$ & $(n=1,347)$ & $(n=1,356)$ & $(n=1,150)$ & $(n=1,117)$ & \\
\hline Age, in years & $56.5(8.6)$ & $59.7(9.3)$ & $63.4(9.3)$ & $67.8(9.6)$ & $<0.0001$ \\
\hline Male & $511(38 \%)$ & $647(48 \%)$ & $621(54 \%)$ & $591(53 \%)$ & $<0.0001$ \\
\hline Female & $836(62 \%)$ & $709(52 \%)$ & $529(46 \%)$ & $526(47 \%)$ & $<0.0001$ \\
\hline Caucasian & $453(33 \%)$ & $505(37 \%)$ & $484(42 \%)$ & $501(45 \%)$ & $<0.0001$ \\
\hline Chinese & $234(17 \%)$ & $172(13 \%)$ & $129(11 \%)$ & $116(10 \%)$ & $<0.0001$ \\
\hline African & $384(29 \%)$ & $359(26 \%)$ & $257(22 \%)$ & $274(25 \%)$ & $<0.003$ \\
\hline Hispanic & $276(20 \%)$ & $320(24 \%)$ & $280(24 \%)$ & $226(20 \%)$ & $<0.024$ \\
\hline \multicolumn{6}{|c|}{ Medical history } \\
\hline HTN & $425(32 \%)$ & $491(36 \%)$ & $524(46 \%)$ & $669(60 \%)$ & $<0.0001$ \\
\hline Diabetes & $142(11 \%)$ & $120(9 \%)$ & $95(8 \%)$ & $155(14 \%)$ & $<0.0001$ \\
\hline Current smokers & $158(12 \%)$ & $165(12 \%)$ & $151(13 \%)$ & $157(14 \%)$ & $<0.31$ \\
\hline Renal disease (self-report) & $20(1.5 \%)$ & $17(1.3 \%)$ & $31(2.7 \%)$ & $45(4.0 \%)$ & 0.0001 \\
\hline \multicolumn{6}{|c|}{ Laboratory/clinical parameters } \\
\hline Creatinine $\mathrm{mg} / \mathrm{dL}$ & $0.84(0.2)$ & $0.91(0.2)$ & $0.97(0.2)$ & $1.13(0.5)$ & $<0.0001$ \\
\hline GFR MDRD mL/min $/ 1.73 \mathrm{~m}^{2}$ & $91.8(16.0)$ & $84.0(14.2)$ & $78.4(13.5)$ & $67.8(16.0)$ & $<0.0001$ \\
\hline Systolic BP, mm Hg & $121.2(20.2)$ & $123.8(20.0)$ & $126.7(21.0)$ & $131.4(22.9)$ & $<0.0001$ \\
\hline Diastolic BP, mm Hg & $71.5(10.5)$ & $72.3(10.2)$ & $72.0(9.9)$ & $71.6(10.6)$ & 0.19 \\
\hline Body mass index $\mathrm{Kg} / \mathrm{m}^{2}$ & $26.7(4.8)$ & $27.5(4.7)$ & $28.2(4.9)$ & $28.8(5.2)$ & $<0.0001$ \\
\hline Body surface area $\mathrm{m}^{2}$ & $1.80(0.2)$ & $1.85(0.2)$ & $1.87(0.2)$ & $1.88(0.2)$ & $<0.0001$ \\
\hline \multicolumn{6}{|c|}{ Medications } \\
\hline Antihypertensive meds & $322(24 \%)$ & $414(31 \%)$ & $421(37 \%)$ & $598(54 \%)$ & $<0.0001$ \\
\hline Beta-blockers & $70(5 \%)$ & $86(6 \%)$ & $110(10 \%)$ & $157(14 \%)$ & $<0.0001$ \\
\hline ACE inhibitors & $107(8 \%)$ & $113(8 \%)$ & $122(11 \%)$ & $199(18 \%)$ & $<0.0001$ \\
\hline $\mathrm{ARB}$ & $23(2 \%)$ & $41(3 \%)$ & $30(3 \%)$ & $67(6 \%)$ & $<0.0001$ \\
\hline Calcium channel blockers & $127(9 \%)$ & $146(11 \%)$ & $145(13 \%)$ & $174(16 \%)$ & $<0.0001$ \\
\hline Thiazide diuretics & $47(3 \%)$ & $70(5 \%)$ & $79(7 \%)$ & $126(11 \%)$ & $<0.0001$ \\
\hline Loop diuretics & $10(1 \%)$ & $11(1 \%)$ & $15(1 \%)$ & $40(4 \%)$ & $<0.0001$ \\
\hline
\end{tabular}

Continuous variables as mean (standard deviation) and categorical variables as percentages; $P$ value obtained using analysis of variance and chi-square test across cystatin C quartiles. cystatin C quartiles $1: \leq 0.76$, quartile $2:>0.76 \& \leq 0.86$, quartile $3:>0.86 \& \leq 0.98$, quartile $4:>0.98$ in mg/dL; HTN: hypertension; GFR: glomerular filtration rate; MDRD: modification of diet in renal disease; ACE: angiotensin-converting enzyme; ARB: angiotensin receptor blocker.

In a separate analysis, where serum creatinine or creatinine-based estimated glomerular filtration rate (eGFR-CR) was added in the model to assess the association of cystatin C quartiles with LV parameters, collinearity diagnostics such as variance inflation factor was performed to avoid unstable parameter estimates. Sensitivity analysis between log transformed cystatin C and LV parameters was also performed. All statistical analyses were performed using JMP Version 8 (SAS Institute Inc., Cary, North Carolina).

\section{Results}

3.1. Baseline Characteristics by Cystatin C Quartiles. Of 6,814 MESA participants, 1,786 did not have MRI measures of LV structure and function, and 58 had no serum cystatin C measures, leaving 4,970 participants who were included at baseline for analysis. Cystatin $\mathrm{C}$ levels ranged from 0.36 to $7.59 \mathrm{mg} / \mathrm{L}$ with a mean value of $0.89 \mathrm{mg} / \mathrm{L}$ and a median of $0.86 \mathrm{mg} / \mathrm{L}$ (interquartile range: 0.76 to $0.98 \mathrm{mg} / \mathrm{L}$ ). For ease of comparability with previous studies, cystatin $\mathrm{C}$ was divided into quartiles: quartile $1(\leq 0.76 \mathrm{mg} / \mathrm{L})$, quartile 2 ( $>0.76$ and $\leq 0.86 \mathrm{mg} / \mathrm{L})$, quartile $3(>0.86$ and $\leq 0.98 \mathrm{mg} / \mathrm{L})$, and quartile $4(>0.98 \mathrm{mg} / \mathrm{L})$. Participants with higher concentrations of cystatin $\mathrm{C}$ were more likely to be older, to have diabetes and hypertension, and to use antihypertensive medications. Those with higher cystatin C levels were less likely to be Chinese or black and had higher body mass indices. As expected, increasing quartiles of cystatin $\mathrm{C}$ were associated with higher levels of creatinine and lower creatinine-based estimated glomerular filtration rates (Table 1).

3.2. Cystatin C Quartiles and LV Parameters. The unadjusted associations and $P$ values for trend between higher cystatin $\mathrm{C}$ quartiles and MRI measures of LV remodeling are demonstrated in Table 2. In a multivariate linear analysis, adjusting for age, gender, race/ethnicity, height, and weight (Model 2), increasing quartiles of cystatin C were significantly associated with LVEDV and concentricity. This association remained after further adjustment for history of diabetes, antidiabetic 
TABLE 2: The association of cystatin C with LV parameters in nested models showing $P$ trend with increasing cystatin $C$ quartiles.

\begin{tabular}{|c|c|c|c|c|c|}
\hline Outcomes variables & Quartile 1 & Quartile 2 & Quartile 3 & Quartile 4 & $P$ trend \\
\hline \multicolumn{6}{|c|}{ Model $1(n=4,970)$} \\
\hline LVEDV, ML & $125.7 \pm 0.8$ & $128.7 \pm 0.9$ & $127.0 \pm 0.9$ & $123.9 \pm 1.0$ & 0.12 \\
\hline LVESV, ML & $39.5 \pm 0.4$ & $41 \pm 0.5$ & $40.2 \pm 0.5$ & $39.2 \pm 0.5$ & 0.55 \\
\hline LVM, Gm & $138.0 \pm 1.0$ & $144.8 \pm 1.1$ & $148.2 \pm 1.2$ & $151.9 \pm 1.2$ & 0.0001 \\
\hline LVM/LVEDV, Gm/ML & $1.11 \pm 0.01$ & $1.14 \pm 0.01$ & $1.19 \pm 0.01$ & $1.25 \pm 0.01$ & 0.0001 \\
\hline $\mathrm{EF}, \%$ & $69.2 \pm 0.2$ & $68.8 \pm 0.2$ & $69.0 \pm 0.2$ & $69.2 \pm 0.2$ & 0.90 \\
\hline \multicolumn{6}{|c|}{ Model $2(n=4,970)$} \\
\hline LVEDV, ML & $128.1 \pm 0.7$ & $127.5 \pm 0.7$ & $125.5 \pm 0.7$ & $124.3 \pm 0.8$ & 0.0002 \\
\hline LVESV, ML & $40.1 \pm 0.4$ & $40.1 \pm 0.4$ & $39.2 \pm 0.4$ & $39.2 \pm 0.5$ & 0.07 \\
\hline LVM, Gm & $145.7 \pm 0.8$ & $145.1 \pm 0.8$ & $144.9 \pm 0.8$ & $148.0 \pm 0.9$ & 0.12 \\
\hline LVM/LVEDV, Gm/ML & $1.15 \pm 0.01$ & $1.15 \pm 0.01$ & $1.17 \pm 0.01$ & $1.21 \pm 0.01$ & 0.0001 \\
\hline $\mathrm{EF}, \%$ & $69.4 \pm 0.2$ & $69.3 \pm 0.2$ & $69.4 \pm 0.2$ & $69.2 \pm 0.2$ & 0.71 \\
\hline \multicolumn{6}{|c|}{ Model $3(n=4,946)$} \\
\hline LVEDV, ML & $128.2 \pm 0.7$ & $127.6 \pm 0.7$ & $125.5 \pm 0.7$ & $124.0 \pm 0.8$ & 0.0001 \\
\hline LVESV, ML & $40.1 \pm 0.4$ & $40.1 \pm 0.4$ & $39.1 \pm 0.4$ & $39.0 \pm 0.5$ & 0.04 \\
\hline LVM, Gm & $146.1 \pm 0.8$ & $145.7 \pm 0.7$ & $145.3 \pm 0.8$ & $147.3 \pm 0.8$ & 0.45 \\
\hline LVM/LVEDV, Gm/ML & $1.15 \pm 0.01$ & $1.16 \pm 0.01$ & $1.17 \pm 0.01$ & $1.21 \pm 0.01$ & 0.0001 \\
\hline $\mathrm{EF}, \%$ & $69.5 \pm 0.2$ & $69.3 \pm 0.2$ & $69.5 \pm 0.2$ & $69.3 \pm 0.2$ & 0.75 \\
\hline
\end{tabular}

Cystatin C quartiles 1: $\leq 0.76$, quartile 2: $>0.76 \& \leq 0.86$, quartile $3:>0.86 \& \leq 0.98$, quartile $4:>0.98 \mathrm{in} \mathrm{mg} / \mathrm{dL}$; LV parameters in adjusted least square mean \pm standard error. LVEDV: LV end diastolic volume; LVESV: LV end systolic volume; LVM: LV mass; LVM/LVEDV: LV concentricity; EF: ejection fraction (fraction).

Model 1 univariate analysis; Model 2 adjusted for age, race/ethnicity, gender, height, and weight; Model 3 adjusted for Model 2 plus diabetes, use of antidiabetic medications, hypertension history, systolic blood pressure, total cholesterol, antilipid medications, and smoking.

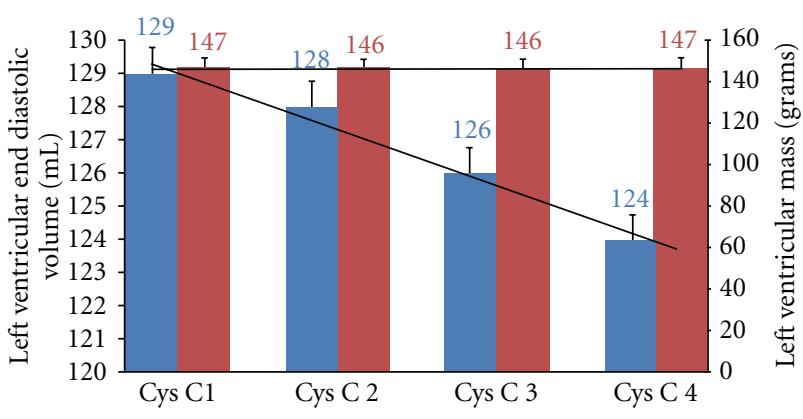

LVEDV in $\mathrm{mL}, P$ trend $=0.0001$

LVM in Gm, $P$ trend $=0.45$

Figure 1: $P$ trend in a fully adjusted model between Cystatin C quartiles and LVEDV and LVM in the MESA cohort (2000-2002). Cystatin C Quartiles 1: $\leq 0.76$, Quartile 2: $>0.76 \& \leq 0.86$, Quartile 3: $>0.86 \& \leq 0.98$, Quartile 4: $>0.98$; LVEDV, LV End Diastolic Volume; LVM, LV Mass; Model adjusted for age, gender, race/ethnicity, height, weight, diabetes, use of anti-diabetic medications, hypertension history, systolic blood pressure, total cholesterol, anti-lipid medications, and smoking.

medications, hypertension, systolic blood pressure, antihypertensive medications, total cholesterol, antilipid medications, and smoking (Model 3) (Figure 1). There was no relationship between cystatin C and LVM or EF in a fully adjusted model (Figure 1). Next, we assessed the influence of race/ethnicity and gender on the associations between cystatin $\mathrm{C}$ quartiles and LV parameters in a fully adjusted model. The associations observed between cystatin C and LV parameters were qualitatively similar within race subgroups and in both genders.

In a separate analysis after further adjustment for serum creatinine, the association between cystatin $C$ quartiles and LV remodeling (LVEDV $P=0.0001$, LVESV $P=0.04$, and LVM/LVEDV $P=0.0001$ ) remained highly significant. The results of collinearity diagnostics revealed a variance inflation factor (VIF) of 1.14 for both creatinine and cystatin C, with creatinine and cystatin $\mathrm{C}$ in the model, and a VIF of 1.37 and 1.47, for creatinine and cystatin $\mathrm{C}$, respectively, in the full model.

In another separate analysis, after further adjustment for creatinine-estimated GFR (eGFR-CR) instead of creatinine in the full model, associations between cystatin $C$ quartiles and LV parameters: $\operatorname{LVEDV}(P=0.0004), \operatorname{LVESV}(P=$ $0.03)$, and $\operatorname{LVM} / \operatorname{LVEDV}(P=0.0001)$ remained highly significant. The results of collinearity diagnostics revealed a variance inflation factor (VIF) of 1.33 for both eGFR-CR and cystatin C, with only eGFR-CR and cystatin C in the model, and a VIF of 1.55 and 1.66, for eGFR-CR and cystatin C, respectively, in the full model. The associations between log transformed cystatin C and LV parameters were similar, and hence data was not presented.

\section{Discussion}

In this large, ethnically diverse population of 4,970 individuals of ages 44-84, free of cardiovascular disease, cystatin $\mathrm{C}$ was inversely associated with LV end-diastolic and 
end-systolic volumes and directly associated with concentricity independent of hypertension, antihypertensive medications, and traditional cardiovascular risk factors. The association observed between cystatin C and LV parameters was not modified by ethnicity or gender. No association was demonstrated between cystatin C and LV end-diastolic mass and LV ejection fraction overall in the population or when stratified by ethnicity and gender.

Ventricular remodeling is a broad term which refers to deviation of normal cardiac structure with notable changes in chamber volumes, wall thickness, and shape [15]. Ventricular remodeling is influenced by the type and duration of cardiovascular injury, and genetic influences [16]. Ventricular remodeling incorporates various morphometric patterns including concentric LV remodeling (increased ratio of LV mass to end-diastolic volume) [17]. Progressive concentric LV remodeling is a key feature of diastolic dysfunction [18], diastolic heart failure [19], and cardiovascular morbidity and mortality [20, 21].

In the Heart and Soul cohort $[4,11]$ with echocardiographic measures of LV structure in approximately 800 participants with coronary artery disease, cystatin $\mathrm{C}$ was associated with LV hypertrophy, diastolic dysfunction, and incident heart failure. However, the relationship between cystatin C and LV volumes was not evaluated. Similarly, Patel et al. [9] in the Dallas Heart Study (DHS) found an association between cystatin $\mathrm{C}$ and LV mass, concentric hypertrophy, and LV wall thickness after adjusting for cardiovascular risk factors in a cohort of 2,500 Caucasians and African-Americans. However, the authors did not find significant association between cystatin C and LV volumes, unlike our study, perhaps reflecting a younger cohort age (DHS, 30 to 65 years versus MESA 44 to 84 years) and/or shorter duration of renal dysfunction. An association between mild-moderate kidney dysfunction and LV hypertrophy was demonstrated in 4,971 MESA participants using cystatin C-derived GFR, but the study did not address the association of kidney dysfunction and LV volumes [10]. The current study extends and corroborates previous findings into a larger multisite multi-ethnic population free of cardiovascular disease by exploring the relationship between cystatin $\mathrm{C}$ and LV volumes and concentricity with more precise measures of cardiac structure and function using magnetic resonance imaging.

Koenig et al. [22] studied 1,033 subjects and found a stronger association of cystatin $\mathrm{C}$ with CVD events compared to creatinine or estimated GFR, suggesting that current estimates of renal function do not fully explain renal dysfunction particularly in mild-to-moderate stages. Similarly, Patel et al. [9] after adjusting for creatinine and GFR in their models demonstrated significant association between cystatin C and concentric hypertrophy. In this study, we adjusted for known risk factors and further adjusted for creatinine or creatinineestimated GFR and found significant association between cystatin C quartiles and LV parameters, which corroborates with such previous findings $[9,22]$.

The mechanisms of association between cystatin $\mathrm{C}$ and cardiovascular disease are not fully characterized, and several complementary hypotheses can be envisioned. Renal insufficiency is a known risk factor for heart failure [23], diastolic dysfunction, and LV remodeling [24]. The risk of cardiovascular remodeling attributable to mild-to-moderate renal insufficiency may not be fully captured by current estimates of renal function such as creatinine, GFR, and creatinine clearance. Thus, as a more precise marker of renal function [6], cystatin $\mathrm{C}$ is associated with ventricular remodeling. Second, cystatin $\mathrm{C}$ is highly correlated with hypertension [9], and the observed association between cystatin C and LV remodeling could be the result of adjustment by imprecise measures of blood pressure leading to residual confounding. Finally, cystatin C may play a direct physiologic role and influence ventricular remodeling independent of hypertension and renal function. The balance between cysteine proteases (cathepsins B, S, and $\mathrm{K}$ ) and cysteine protease inhibitors (cystatin C) has been implicated in pathological LV remodeling in heart failure $[25,26]$. Cathepsins, stored in lysosomes, are responsible for the physiological digestive turnover of cellular molecules, and abnormal levels of which may adversely influence cardiac remodeling [26]. Although the mechanism between cystatin C and LV remodeling is unclear, it is possible that the alteration of the balance between these two classes of proteins may contribute to the LV remodeling process by matrix degradation [26].

The strength of this study includes a large ethnically diverse population with stringent quality control procedures and MRI measurements of cardiac structure and function. The present study extends prior investigations and supports the view that kidney disease approximated by elevated cystatin $\mathrm{C}$ is associated with concentric remodeling. We found modest changes in LV volumes across higher quartiles of cystatin $\mathrm{C}$ after adjustment which was found to be statistically significant. The clinical implications of these findings are not yet clear. It remains to be seen whether the pattern of LV remodeling demonstrated in the current study is associated with subsequent development of clinical heart failure or cardiovascular mortality in the MESA cohort.

We acknowledge several limitations. MESA did not directly measure glomerular filtration rate; therefore, we cannot be certain that the association between elevated cystatin C level and LV parameters are solely caused by its approximation of impaired GFR. Due to the cross-sectional design, the study cannot address the direction of the association between cystatin C and LV remodeling, and the observed associations could be purely a statistical phenomenon; however, the results are consistent with previous such observed relationships which point towards a true association. The association observed between cystatin C and LV remodeling could result from decreased renal perfusion from a reduced cardiac output, or structural remodeling of arteries, although these possibilities were minimized by inclusion of a cardiovascular disease-free population, though self-reported history may be imperfect due to recall bias. Although efforts were made to adjust for known confounders, there remains a possibility of failure to adjust for unknown confounders or inadequate adjustment of established risk factors (severity and duration of hypertension, diabetes) resulting in spurious results due to residual confounding. Some studies suggest that corticosteroids [27] and thyroid function [28] are associated with cystatin $\mathrm{C}$, and since adjustment with these measures was not 
performed, results should be interpreted with caution in this subset of individuals.

\section{Conclusion}

Cystatin C levels are inversely associated with LV end diastolic and systolic volumes and directly associated with concentricity independent of traditional cardiovascular risk factors including hypertension in a multi-ethnic population. This morphometric pattern of concentric LV remodeling may in part explain previously observed associations between cystatin $\mathrm{C}$ and diastolic dysfunction, heart failure, and cardiovascular mortality.

\section{Disclosures}

The authors had full access to the data and take responsibility for the integrity of the data. All the authors have read and agreed to the manuscript as written. This research was supported by NHLBI, NIH T32 training Grant (no.T32 HL 076132-06 A1), and Contracts nos. N01-HC95159 through N01-HC-95169 from the National Heart, Lung, and Blood Institute. The authors thank the other investigators, the staff, and the participants of the MESA study for their valuable contributions. A full list of participating MESA investigators and institutions can be found at http://www.mesa-nhlbi.org/.

\section{References}

[1] M. G. Shlipak, M. J. Sarnak, R. Katz et al., "Cystatin C and the risk of death and cardiovascular events among elderly persons," New England Journal of Medicine, vol. 352, no. 20, pp. 2049-2060, 2005.

[2] M. J. Sarnak, R. Katz, C. O. Stehman-Breen et al., "Cystatin C concentration as a risk factor for heart failure in older adults," Annals of Internal Medicine, vol. 142, no. 7, pp. 497-505, 2005.

[3] M. G. Shlipak, R. Katz, L. F. Fried et al., "Cystatin-C and mortality in elderly persons with heart failure," Journal of the American College of Cardiology, vol. 45, no. 2, pp. 268-271, 2005.

[4] J. H. Ix, M. G. Shlipak, G. M. Chertow, and M. A. Whooley, "Association of cystatin C with mortality, cardiovascular events, and incident heart failure among persons with coronary heart disease: data from the Heart and Soul Study," Circulation, vol. 115, no. 2, pp. 173-179, 2007.

[5] J. Kyhse-Andersen, C. Schmidt, G. Nordin et al., "Serum cystatin $\mathrm{C}$, determined by a rapid, automated particle-enhanced turbidimetric method, is a better marker than serum creatinine for glomerular filtration rate," Clinical Chemistry, vol. 40, no. 10, pp. 1921-1926, 1994.

[6] D. J. Newman, H. Thakkar, R. G. Edwards et al., "Serum cystatin $\mathrm{C}$ measured by automated immunoassay: a more sensitive marker of changes in GFR than serum creatinine," Kidney International, vol. 47, no. 1, pp. 312-318, 1995.

[7] I. Helin, M. Axenram, and A. Grubb, "Serum cystatin C as a determinant of glomerular filtration rate in children," Clinical Nephrology, vol. 49, no. 4, pp. 221-225, 1998.

[8] E. Coll, A. Botey, L. Alvarez et al., "Serum cystatin C as a new marker for noninvasive estimation of glomerular filtration rate and as a marker for early renal impairment," American Journal of Kidney Diseases, vol. 36, no. 1, pp. 29-34, 2000.
[9] P. C. Patel, C. R. Ayers, S. A. Murphy et al., "Association of cystatin $\mathrm{C}$ with left ventricular structure and function : the Dallas heart study," Circulation: Heart Failure, vol. 2, no. 2, pp. 98-104, 2009.

[10] A. Moran, R. Katz, N. S. Jenny et al., "Left ventricular hypertrophy in mild and moderate reduction in kidney function determined using cardiac magnetic resonance imaging and cystatin C: the multi-ethnic study of atherosclerosis (MESA)," American Journal of Kidney Diseases, vol. 52, no. 5, pp. 839$848,2008$.

[11] J. H. Ix, M. G. Shlipak, G. M. Chertow et al., "Left ventricular hypertrophy, and diastolic dysfunction: data from the Heart and Soul Study," Journal of Cardiac Failure, vol. 12, no. 8, pp. 601-607, 2006.

[12] D. E. Bild, D. A. Bluemke, G. L. Burke et al., "Multi-ethnic study of atherosclerosis: objectives and design," American Journal of Epidemiology, vol. 156, no. 9, pp. 871-881, 2002.

[13] E. J. Erlandsen, E. Randers, and J. H. Kristensen, "Evaluation of the dade behring $\mathrm{N}$ latex cystatin $\mathrm{C}$ assay on the dade behring nephelometer II system," Scandinavian Journal of Clinical and Laboratory Investigation, vol. 59, no. 1, pp. 1-8, 1999.

[14] S. Natori, S. Lai, J. P. Finn et al., "Cardiovascular function in multi-ethnic study of atherosclerosis: normal values by age, sex, and ethnicity," American Journal of Roentgenology, vol. 186, supplement 2, no. 6, pp. S357-S365, 2006.

[15] M. A. Konstam, J. E. Udelson, I. S. Anand, and J. N. Cohn, "Ventricular remodeling in heart failure: a credible surrogate endpoint," Journal of Cardiac Failure, vol. 9, no. 5, pp. 350353, 2003.

[16] G. Doolan, L. Nguyen, J. Chung, J. Ingles, and C. Semsarian, "Progression of left ventricular hypertrophy and the angiotensin-converting enzyme gene polymorphism in hypertrophic cardiomyopathy," International Journal of Cardiology, vol. 96, no. 2, pp. 157-163, 2004.

[17] M. G. Khouri, R. M. Peshock, C. R. Ayers, J. A. de Lemos, and M. H. Drazner, "A 4-tiered classification of left ventricular hypertrophy based on Left ventricular geometry: the Dallas Heart study," Circulation: Cardiovascular Imaging, vol. 3, no. 2, pp. 164-171, 2010.

[18] S. Lalande and B. D. Johnson, "Diastolic dysfunction: a link between hypertension and heart failure," Drugs of Today, vol. 44, no. 7, pp. 503-513, 2008.

[19] J. N. Cohn, R. Ferrari, and N. Sharpe, "Cardiac remodelingconcepts and clinical implications: a consensus paper from an international forum on cardiac remodeling. Behalf of an International Forum on Cardiac Remodeling," Journal of the American College of Cardiology, vol. 35, no. 3, pp. 569-582, 2000.

[20] D. A. Bluemke, R. A. Kronmal, J. A. C. Lima et al., "The relationship of left ventricular mass and geometry to incident cardiovascular events: the MESA (Multi-Ethnic Study of Atherosclerosis) study," Journal of the American College of Cardiology, vol. 52, no. 25, pp. 2148-2155, 2008.

[21] P. Verdecchia, G. Schillaci, C. Borgioni et al., "Adverse prognostic significance of concentric remodeling of the left ventricle in hypertensive patients with normal left ventricular mass," Journal of the American College of Cardiology, vol. 25, no. 4, pp. 871-878, 1995.

[22] W. Koenig, D. Twardella, H. Brenner, and D. Rothenbacher, "Plasma concentrations of cystatin $\mathrm{C}$ in patients with coronary heart disease and risk for secondary cardiovascular events: more than simply a marker of glomerular filtration rate," Clinical Chemistry, vol. 51, no. 2, pp. 321-327, 2005. 
[23] A. S. Go, G. M. Chertow, D. Fan, C. E. McCulloch, and C. Y. $\mathrm{Hsu}$, "Chronic kidney disease and the risks of death, cardiovascular events, and hospitalization," New England Journal of Medicine, vol. 351, no. 13, pp. 1296-1305, 2004.

[24] Z. Dimitrijevic, T. Cvetkovic, M. Stojanovic, K. Paunovic, and V. Djordjevic, "Prevalence and risk factors of myocardial remodeling in hemodialysis patients," Renal Failure, vol. 31, no. 8, pp. 662-667, 2009.

[25] X. W. Cheng, K. Obata, M. Kuzuya et al., "Elastolytic cathepsin induction/activation system exists in myocardium and is upregulated in hypertensive heart failure," Hypertension, vol. 48, no. 5, pp. 979-987, 2006.

[26] F. Sam and D. A. Siwik, "Digesting the remodeled heart: role of lysosomal cysteine proteases in heart failure," Hypertension, vol. 48, no. 5, pp. 830-831, 2006.

[27] L. Risch, R. Herklotz, A. Blumberg, and A. R. Huber, "Effects of glucocorticoid immunosuppression on serum cystatin $\mathrm{C}$ concentrations in renal transplant patients," Clinical Chemistry, vol. 47, no. 11, pp. 2055-2059, 2001.

[28] P. Wiesli, B. Schwegler, G. A. Spinas, and C. Schmid, "Serum cystatin C is sensitive to small changes in thyroid function," Clinica Chimica Acta, vol. 338, no. 1-2, pp. 87-90, 2003. 


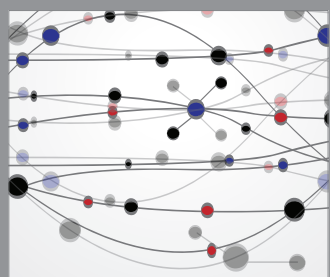

The Scientific World Journal
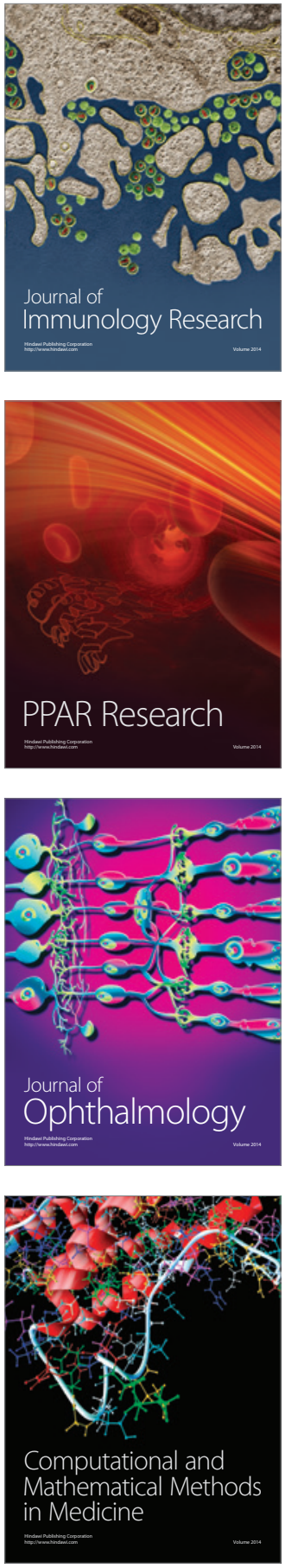

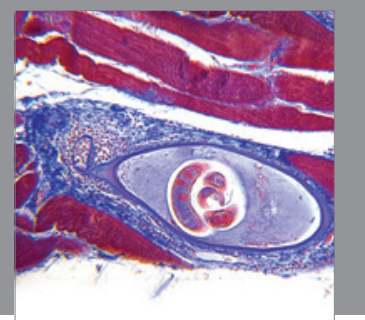

Gastroenterology

Research and Practice
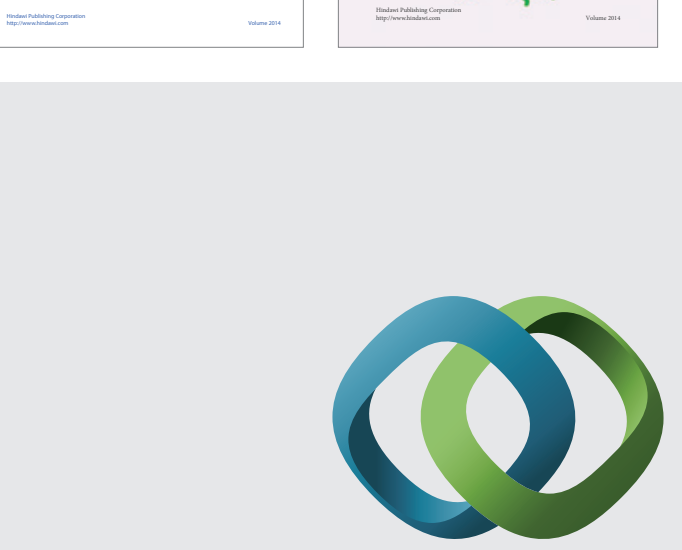

\section{Hindawi}

Submit your manuscripts at

http://www.hindawi.com
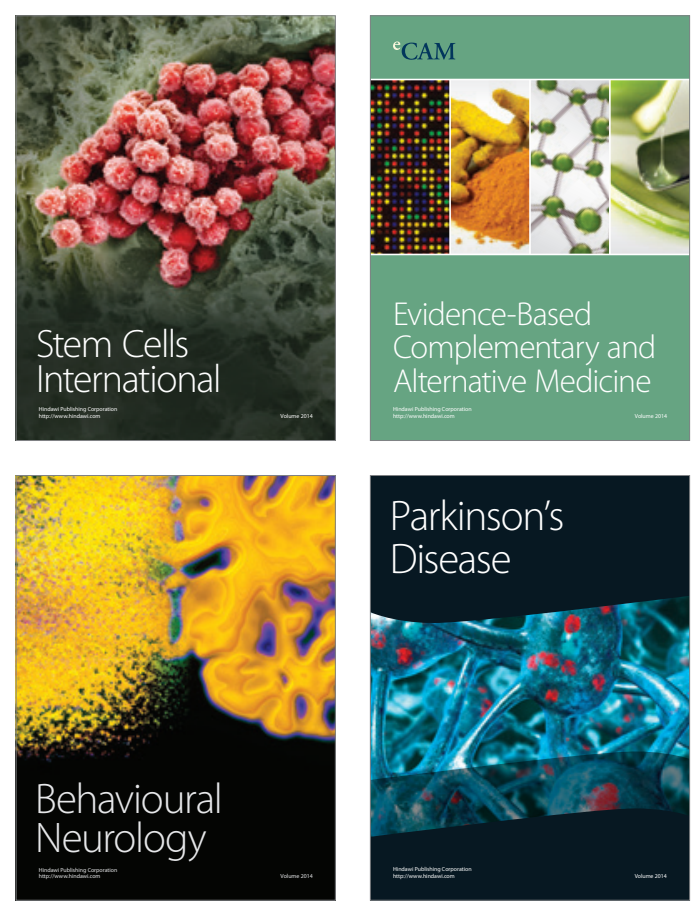

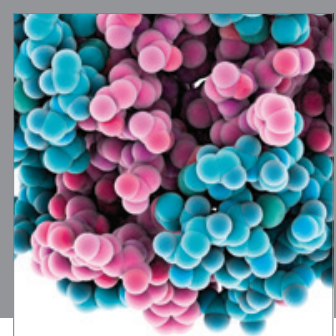

Journal of
Diabetes Research

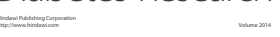

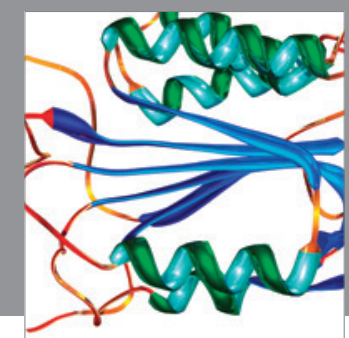

Disease Markers
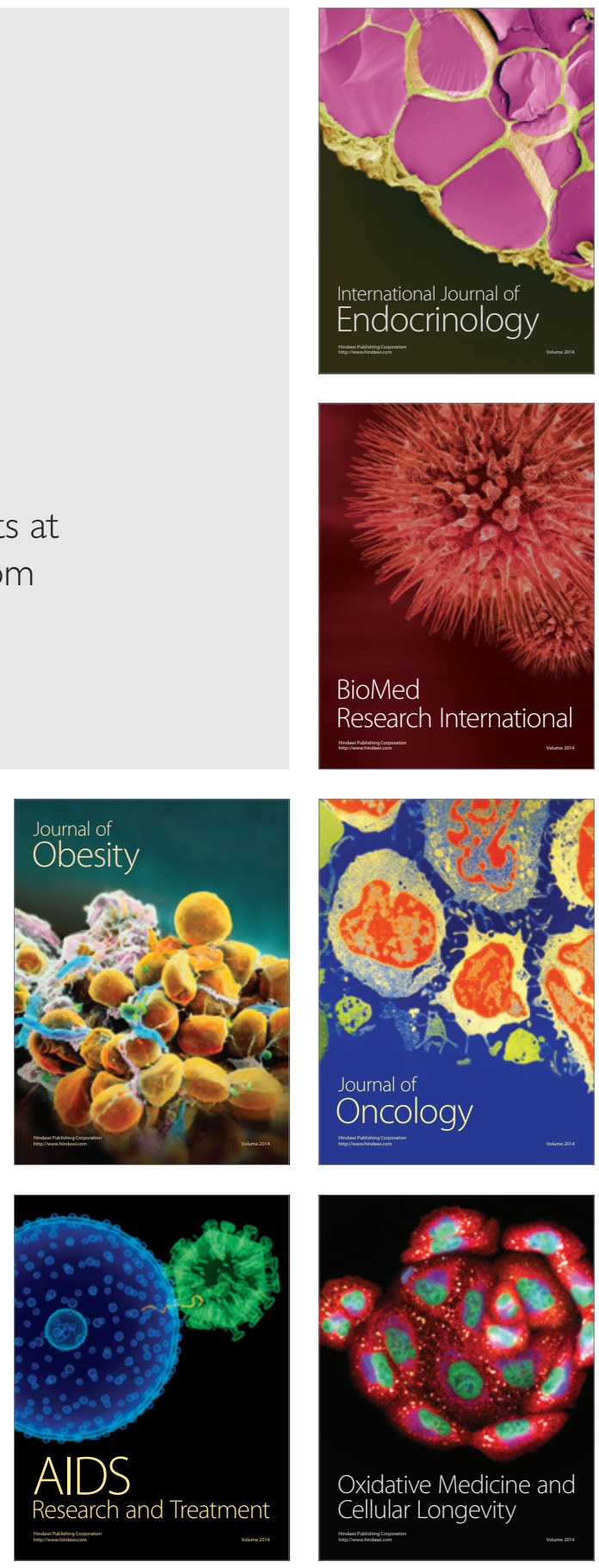